\title{
Health Risk Analysis of Indoor Air Pollution
}

\author{
K. F. R. Liu, K. Yeh, M.-J. Hung, C.-W. Chen, and Y.-S. Shen
}

\begin{abstract}
This study uses target organ-specific hazard index (TOSHI) and cancer Risk to analyze the health risk for indoor air pollutants defined in Taiwan Indoor Air Quality Management Act. The reference concentrations (RfC) refer to the minimal values among chronic reference exposure level (REL) developed by OEHHA, guideline values developed by WHO, threshold limit values (TLV) developed by ACGIH, and Taiwan Indoor Air Quality Standard. As for cancer unit risk, the minimal values between OEHH and WHO are considered. Finally, the method is performed to analyze the health risk of IAQ in a local hospital, before and after improvement plan, respectively.
\end{abstract}

Index Terms - Indoor air quality, health risk analysis, hazard index, reference concentration, cancer risk, unit risk.

\section{INTRODUCTION}

The Taiwan Indoor Air Quality Act [1] is formulated to improve indoor air quality and to protect public health. Indoor air pollutant means substances that are normally dispersed in indoor air, and which may directly or indirectly affect public health or the living environment after long-term exposure, including carbon dioxide $\left(\mathrm{CO}_{2}\right)$, carbon monoxide $(\mathrm{CO})$, formaldehyde( $\mathrm{HCHO}$ ), total volatile organic compounds (TVOC), bacteria, fungi, airborne particles with a particle diameter of 10 micrometers or less $\left(\mathrm{PM}_{10}\right)$, airborne particles with a diameter of 2.5 micrometers or less $\left(\mathrm{PM}_{2.5}\right)$ and ozone $\left(\mathrm{O}_{3}\right)$.Indoor air quality has become a topic of interest and concern, considering the increased number of reported 'sick building's yndrome and 'building-related' illness cases where office workers complain of exposures to contaminants in the air. Symptoms include fatigue, coughs, upper respiratory diseases, headaches and dizziness. The possible health effect due to the indoor air pollutants are discussed as follows.

Carbon dioxide in low concentration is non-toxic, while its high concentration will cause human choking effect. Currently, the observed minimal concentration affecting human health is $7,000 \mathrm{ppm}$, and a continued exposure to this concentration will lower a person the $\mathrm{PH}$ value in his blood. After prolonged exposure (days) to $3,500 \mathrm{ppm}$, acid-base

Manuscript received April 5, 2014; revised July 24, 2014

K. F. R. Liu and M. J. Hung are with the Department of Safety, Health and Environmental Engineering, Ming Chi University of Technology, New Taipei City, Taiwan 24301, ROC (e-mail: kevinliu@mail.mcut.edu.tw, mingjui@mail.mcut.edu.tw).

$\mathrm{K}$. Yeh is with the Department of Construction Science and Technology, De-Lin Institute of Technology, New Taipei City, Taiwan 23654, ROC (e-mail: kyeh@dlit.edu.tw).

C. W. Chen is with the Institute of Maritime Information and Technology, National Kaohsiung Marine University, Kaohsiung, Taiwan 80543, ROC (e-mail: chengwu@webmail.nkmu.edu.tw).

Y. S. Shen is with the Holistic Education Center, Mackay Medical College, New Taipei City, Taiwan 25245, ROC (e-mail: ysshen@mmc.edu.tw). regulation can occur via renal mechanisms which can affect calcium metabolism in bone. Carbon dioxide currently is viewed as an indicator for ventilation because when its concentration is higher than $1,000 \mathrm{ppm}$, other indoor pollutants will be monitored. Poor ventilation can make indoor environment uncomfortable and can reduce productivity. Poor ventilation can also lead to increased humidity, as moisture produced indoors is not vented to the outside. High humidity can encourage the growth of mold and dust mites; both of which are allergens and asthma triggers. In addition, ventilation also helps reduce the levels of other indoor air pollutants released from furnishings, building products or chemical cleaners such as formaldehyde or volatile organic compounds (VOCs). Since some of these chemicals have known or suspected health effects, keeping levels as low as possible is always advisable.

Carbon monoxide is a colorless, odorless, toxic gas even at low concentration. It occurs where combustion gases are not properly exhausted or are being re-entrained into the building. In office and commercial buildings, important sources of combustion contaminants include tobacco smoke, garages, and loading docks that are attached or have a pathway to working spaces. Air intakes located at ground level or adjacent to vehicles or other combustion sources can transport contaminants to areas served by the air handling system. Carbon monoxide is extremely toxic. It combines with hemoglobin in the blood, reducing the oxygen supply to the body. At elevated levels, symptoms of exposure include headaches, decreased alertness, flu-like symptoms, nausea, fatigue, rapid breathing, chest pain, confusion, and impaired judgment.

Formaldehyde is a colorless gas. A pungent odor often indicates its presence at a concentration greater than $0.2 \mathrm{ppm}$. Formaldehyde is present when vapors off-gas from building materials (e.g., carpets, particleboard, fabrics), cleaning fluids, and adhesives. Indoor concentrations are dependent on the age of the source, ventilation rate, indoor and outdoor temperatures, and humidity. Formaldehyde is a known irritant and sensitizer. Symptoms include dry or sore throat, nosebleeds, headaches, fatigue, memory and concentration problems, nausea, dizziness, breathlessness, and burning, stinging, and pain in the eyes. Irritant effects have been associated with concentrations in the median range of 0.5 $\mathrm{ppm}$, and concentrations as low as $0.01 \mathrm{ppm}$ have been reported to affect sensitive individuals. Besides, according to the International Agency for Research of Cancer (IARC), formaldehyde is classified in Group B1 carcinogen. Animal experiments showed that formaldehyde increased incidence of nasopharyngeal carcinoma.

In related laws of Taiwan, total volatile organic compounds includes benzene, carbon tetrachloride, chloroform, 1,2-dichlorobenzene, 1,4-dichlorobenzene, dichloromethane, ethyl benzene, styrene, tetrachloroethylene, 
trichloroethylene, toluene and xylenes. Health hazards of volatile organic compounds include carcinogenic and non-carcinogenic effects. In terms of non-carcinogenic, they will cause damage on liver or kidney systems, and cause respiratory irritation or discomfort. In the IARC classification, benzene is classified in Group and toluene is classified in Group D. But studies showed that a healthy adult can cause eye and nasal irritation if he exposes $100 \mathrm{ppm}$ of toluene up to 6 hours.

Bacteria in indoor environment can be distinguished by shape (cocci, bacilli and spiral body, etc.) and Gram staining. Gram-negative bacteria infection usually causes fever, weakness, pain and shock, probably causing more serious diseases such as typhoid fever, urinary tract infections and meningitis. Gram-positive bacteria will affect the structure or function of specific cells, causing diseases such as gas gangrene, tetanus, botulism, diphtheria and scarlet fever, and the like.

Fungi can cause human allergies, infection and toxicity. Common allergic reactions are allergic asthma and allergic rhinitis. Repeated exposure to high concentrations of the fungus may cause hypersensitivity pneumonitis. Fungal infection often occurs in the skin and mucosal surfaces. Deep tissue fungal infection is usually confined to patients with severe defects in immune function, such as diabetes and AIDS patients. In addition to infection and allergy, part of fungal metabolites can be toxic, such as mycotoxins.

The damage of human respiratory tract by suspended particulate relies on three factors, chemical composition, penetration and sedimentary position. Penetration and sedimentary position depend on particle size. According to International Radiological Protection Commission, the diameter at $10 \sim 100 \mu \mathrm{m}$ of particles will sediment in human nasal; particles with diameter less than $10 \mu \mathrm{m}\left(\mathrm{PM}_{10}\right)$ almost sediment in alveolar and airway; particles with diameter less than $2.5 \mu \mathrm{m}\left(\mathrm{PM}_{2.5}\right)$ sediment in the lungs in the highest efficiency. Suspended particulates in the alveoli and trachea will result in allergic rhinitis, asthma, chronic obstructive pulmonary disease and other diseases.

Indoor ozone comes from copiers, printers and electrostatic air cleaning devices. Damage caused by ozone on respiratory tract can be divided into two types, one is the change in lung function and the accompanied respiratory symptoms; two is the damage to the structures or functions of special cells.

According to the definition of target organs or systems by The California Office of Environmental Health Hazard Assessment (OEHHA) [2], the health effects due to indoor air pollutants are summarized in Table I.

\section{METHODS AND MATERIALS}

Health risk assessment (HRA) is the process for estimating the nature and probability of adverse health effects in humans who may be exposed to hazardous substances. Its four basic ingredients include hazard identification, exposure assessment, dose-response assessment, and risk characterization [3]. Hazard identification aims to recognize any potential health problem that a substance can cause; exposure assessment determines the amount, duration, and pattern of exposure to the substance; dose-response assessment estimates how much of the substance it would take to cause varying degrees of adverse health effects; and risk characterization interprets the risk for the substance to cause cancer or other illnesses. In risk characterization of non-carcinogenic substances, the HRA can be evaluated by the hazard quotient (HQ), the ratio of the intake of a hazardous substance to its reference dose. Multiple hazardous substances may affect the same organ (or organ system) causing joint effect; and hence, the target organ-specific hazard index (TOSHI) sums the HQ scores of multiple substances that have joint effect on a specific organ [4].

TABlE I: The Health EfFects Due to Indoor Air Pollutants

\begin{tabular}{|c|c|}
\hline Indoor air pollutant & Affected target organ or system ${ }^{\mathrm{a}}$ \\
\hline $\mathrm{CO}_{2}$ & $\mathrm{Na}^{\mathrm{b}}$ \\
\hline $\mathrm{CO}$ & Cardiovascular system $^{\mathrm{c}}$ \\
\hline $\mathrm{HCHO}$ & Respiratory system $^{\mathrm{a}}$ \\
\hline TVOC & $\mathrm{Na}^{\mathrm{b}}$ \\
\hline Benzene & $\begin{array}{l}\text { Hematological system }^{\text {a }} \\
\text { Nervous system }^{\text {a }} \\
\text { Developmental system }^{\text {a }}\end{array}$ \\
\hline Carbon tetrachloride & $\begin{array}{l}\text { Alimentary system }(\text { Liver })^{\mathrm{a}} \text {; } \\
\text { Reproductive system }\end{array}$ \\
\hline Chloroform & $\begin{array}{l}\text { Alimentary system }{ }^{\mathrm{a}} ; \text { Kidney }^{\mathrm{a}} \text {; } \\
\text { Developmental system }^{\mathrm{a}}\end{array}$ \\
\hline 1,2-Dichlorobenzene & $\mathrm{Na}^{\mathrm{b}}$ \\
\hline 1,4-Dichlorobenzene & 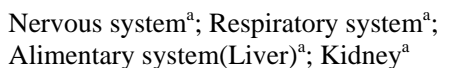 \\
\hline Dichloromethane & $\begin{array}{l}\text { Alimentary system(Liver) }{ }^{\mathrm{a}} ; \text { Kidney }^{\mathrm{a}} ; \\
\text { Nervous system }^{\mathrm{a}} ; \text { Cardiovascular system }\end{array}$ \\
\hline Ethyl Benzene & $\begin{array}{l}\text { Alimentary system(Liver })^{\text {a }} ; \text { Kidney }^{\text {a }} \\
\text { Endocrine system }^{\text {a }} \text {; } \\
\text { Developmental system }^{\text {a }}\end{array}$ \\
\hline Styrene & Nervous system $^{\mathrm{a}}$ \\
\hline Tetrachloroethylene & Kidney $^{\mathrm{a}}$; Alimentary System $(\text { Liver })^{\mathrm{a}}$ \\
\hline Trichloroethylene & Nervous system ${ }^{\mathrm{a}} ;$ Eyes $^{\mathrm{a}}$ \\
\hline Toluene & 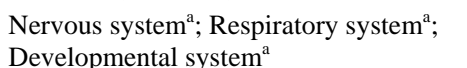 \\
\hline Xylenes & $\begin{array}{l}\text { Nervous system }{ }^{\mathrm{a}} ; \text { Respiratory system }{ }^{\mathrm{a}} \text {; } \\
\text { Eyes }^{\mathrm{a}}\end{array}$ \\
\hline Bacteria & Allergy; Infection ${ }^{\mathrm{c}}$ \\
\hline Fungi & Allergy; Infection ${ }^{\mathrm{c}}$ \\
\hline $\mathrm{PM}_{10}$ & Respiratory system ${ }^{\mathrm{c}}$ \\
\hline $\mathrm{PM}_{2.5}$ & Cardiovascular system ${ }^{\mathrm{c}}$ \\
\hline $\mathrm{O}_{3}$ & Respiratory system $^{\mathrm{a}}$; Eyes ${ }^{\mathrm{a}}$ \\
\hline
\end{tabular}

\section{A. Cancer Risk}

Cancer risk is often expressed as the maximum number of new cases of cancer projected to occur in a population of one million people due to exposure to the cancer-causing substance over a 70-year lifetime [5]. For carcinogens, risks are estimated as the incremental probability of an individual developing cancer over a lifetime as a result of exposure to the potential carcinogen (i.e., incremental or excess individual lifetime cancer risk). The slope factor (SF) converts estimated daily intakes averaged over a lifetime of exposure directly to incremental risk of an individual developing cancer. It generally can be assumed that the dose-response relationship will be linear in the low-dose portion of the multistage model dose-response curve.

$$
\begin{aligned}
& \text { Risk }=\text { Exposure dose } \times \text { slope factor } \\
& =\frac{\mathrm{C} \times \mathrm{IR} \times \mathrm{AT} \times \mathrm{AF}}{\mathrm{BW}}(\mathrm{mg} / \mathrm{kg} / \text { day }) \times \text { slope factor }
\end{aligned}
$$


where $\mathrm{C}$ is a measurement concentration $\left(\mathrm{mg} / \mathrm{m}^{3}\right)$; IR indicates intake $\operatorname{rate}\left(\mathrm{m}^{3} /\right.$ hour $) ;$ AT indicates average time(hour/day); AF indicates absorption fraction(\%); BW indicates body weight $(\mathrm{kg})$; slope factor: $\left(\mu \mathrm{g} / \mathrm{m}^{3}\right)^{-1}$.

If the exposure pathway is inhalation cancer risk can be repressed as

$$
\text { Risk }=\mathrm{C} \times \text { Unit Risk }
$$

where $\mathrm{C}$ is a contaminant concentration $\left(\mathrm{mg} / \mathrm{m}^{3}\right)$; Unit Risk is a unit of risk $\left(\mu \mathrm{g} / \mathrm{m}^{3}\right)^{-1}$.

Generally speaking, the acceptable lifetime cancer risk ranges from one in ten thousand to one in one million. This range may be expressed as $1 \times 10^{-4}$ to $1 \times 10^{-6}$.

\section{B. Non-Cancer Risk}

Non-cancer risk is usually determined by comparing the actual level of exposure to a chemical to the level of exposure that is not expected to cause any adverse effects, even in the most susceptible people [5]. The level of concern for non-carcinogenic contaminants is determined by calculating a Hazard Quotient (HQ) or Hazard Index (HI). An HI is the sum of the HQs for several chemicals that affect the same target organ. If the HQ or HI equals or exceeds one, there may be concern for potential exposure to site contaminants.

$$
\mathrm{HQ}=\frac{\mathrm{C}}{\mathrm{RfC}}
$$

where $\mathrm{C}$ indicates exposure concentration $\left(\mu \mathrm{g} / \mathrm{m}^{3}\right)$; RfC indicates reference concentration $\left(\mu \mathrm{g} / \mathrm{m}^{3}\right)$.

$$
\mathrm{HI}=\Sigma_{i} \mathrm{HQ}_{i}=\Sigma_{i} \frac{\mathrm{C}_{i}}{\mathrm{RfC}_{i}}
$$

where $C_{i}$ indicates the exposure concentration of the $i^{\text {th }}$ substance $\left(\mu \mathrm{g} / \mathrm{m}^{3}\right)$; RfC indicates the reference concentration of the $i^{\text {th }}$ substance $\left(\mu \mathrm{g} / \mathrm{m}^{3}\right)$.

\section{RESULTS}

\section{A. Determination of RfC}

The RfCs of indoor air pollutants primarily refer to the chronic reference exposure level (REL) defined by OEHHA [2], the threshold limit values (TLV) defined by The American Conference of Governmental Industrial Hygienists (ACGIH) [6], the guideline values defined by The World Health Organization (WHO) [7], and the Taiwan Indoor quality Standard [1], as shown in Table II.

\section{B. Calculation of Health Risk}

\section{Cancer risk:}

\begin{tabular}{|c|c|c|c|c|c|c|c|c|c|c|}
\hline \multirow{2}{*}{$\begin{array}{l}\text { Indoor air pollutant } \\
\mathrm{CO}_{2}\end{array}$} & \multicolumn{2}{|c|}{$\begin{array}{c}\text { Taiwan } \\
\text { Indoor Air } \\
\text { Standard }\end{array}$} & \multirow{2}{*}{$\begin{array}{l}\text { Unit } \\
\mathrm{ppm}\end{array}$} & \multirow{2}{*}{$\begin{array}{c}\text { ACGIH } \\
\text { TLV } \\
5,000\end{array}$} & \multirow[t]{2}{*}{$\begin{array}{c}\text { OEHHA } \\
\text { Chronic } \\
\text { REL (ppm) }\end{array}$} & \multirow[t]{2}{*}{$\begin{array}{l}\text { OEHHA Unit } \\
\text { Risk (ppm) })^{-1}\end{array}$} & \multirow[t]{2}{*}{$\begin{array}{c}\text { WHO } \\
\text { Guideline } \\
\text { Value for } \\
\text { Non-Cancer } \\
\text { (ppm) }\end{array}$} & \multirow[t]{2}{*}{$\begin{array}{l}\text { WHO } \\
\text { Guideline } \\
\text { Value for } \\
\text { Cancer } \\
(\mathrm{ppm})^{-1}\end{array}$} & \multirow{2}{*}{$\begin{array}{c}\text { RfC } \\
5,000\end{array}$} & \multirow[t]{2}{*}{$\begin{array}{l}\text { Unit Risk } \\
(\mathrm{ppm})^{-1}\end{array}$} \\
\hline & $8-h$ & 1,000 & & & & & & & & \\
\hline $\mathrm{CO}$ & $8-h$ & 9 & ppm & 25 & & & 10 & & 9 & \\
\hline $\mathrm{HCHO}$ & $1-\mathrm{h}$ & 0.08 & ppm & 0.3 & 0.007328653 & 7.37E-03 & 0.081429482 & & 0.007328653 & 7.37E-03 \\
\hline TVOC & $1-\mathrm{h}$ & 0.56 & ppm & & & & & & 0.56 & \\
\hline Benzene & & & & & 0.018780764 & $9.26 \mathrm{E}-02$ & & $1.92 \mathrm{E}-02$ & 0.018780764 & $1.92 \mathrm{E}-02$ \\
\hline Carbon tetrachloride & & & & & 0.006357969 & 2.64E-01 & & & 0.006357969 & $2.64 \mathrm{E}-01$ \\
\hline Chloroform & & & & & 0.061443667 & $2.59 \mathrm{E}-02$ & & & 0.061443667 & $2.59 \mathrm{E}-02$ \\
\hline \multicolumn{11}{|l|}{ 1,2-Dichlorobenzene } \\
\hline 1,4-Dichlorobenzene & & & & & 0.13305945 & $6.61 \mathrm{E}-02$ & & & 0.13305945 & $6.61 \mathrm{E}-02$ \\
\hline Dichloromethane & & & & & 0.172725237 & $3.47 \mathrm{E}-05$ & 0.863626184 & & 0.172725237 & 3.47E-05 \\
\hline Ethyl Benzene & & & & & 0.460603777 & 1.09E-02 & & & 0.460603777 & $1.09 \mathrm{E}-02$ \\
\hline Styrene & & & & & 0.21128359 & & 0.061037482 & & 0.061037482 & \\
\hline Tetrachloroethylene & & & & & 0.005160299 & $1.76 \mathrm{E}-03$ & 0.036859282 & & 0.036859282 & $1.76 \mathrm{E}-03$ \\
\hline Trichloroethylene & & & & & 0.111653743 & $1.07 \mathrm{E}-02$ & & & 0.111653743 & $1.07 \mathrm{E}-02$ \\
\hline Toluene & & & & & 0.079608485 & & 0.06899402 & & 0.079608485 & \\
\hline Xylenes & & & & & 0.053737107 & & & & 0.053737107 & \\
\hline Bacteria & $\operatorname{Max}$ & 1,500 & $\mathrm{CFU} / \mathrm{m}^{3}$ & & & & & & 1,500 & \\
\hline Fungi & $\operatorname{Max}$ & 1,000 & $\mathrm{CFU} / \mathrm{m}^{3}$ & & & & & & 1,000 & \\
\hline $\mathrm{PM}_{10}$ & 24-h & 75 & $\mu \mathrm{g} / \mathrm{m}^{3}$ & 3000 & & & & & 75 & \\
\hline $\mathrm{PM}_{2.5}$ & 24-h & 35 & $\mu \mathrm{g} / \mathrm{m}^{3}$ & 10000 & & & & & 35 & \\
\hline $\mathrm{O}_{3}$ & $8-\mathrm{h}$ & 0.06 & ppm & 0.2 & 0.091690938 & & 0.061127292 & & 0.06 & \\
\hline
\end{tabular}

$$
\begin{aligned}
\text { Risk }= & \mathrm{C}_{\text {Formaldehyde }}(\mathrm{ppm}) \times 7.37 \mathrm{E}-03(\mathrm{ppm})^{-1} \\
& +\mathrm{C}_{\text {Benzene }}(\mathrm{ppm}) \times 1.92 \mathrm{E}-02(\mathrm{ppm})^{-1} \\
& +\mathrm{C}_{\text {Carbon tetrachloride }}(\mathrm{ppm}) \times 2.64 \mathrm{E}-01(\mathrm{ppm})^{-1} \\
& +\mathrm{C}_{\text {Chloroform }}(\mathrm{ppm}) \times 2.59 \mathrm{E}-02(\mathrm{ppm})^{-1} \\
& +\mathrm{C}_{1,4-\text { Dichlorobenzene }}(\mathrm{ppm}) \times 6.61 \mathrm{E}-02(\mathrm{ppm})^{-1} \\
& +\mathrm{C}_{\text {Dichloromethane }}(\mathrm{ppm}) \times 3.47 \mathrm{E}-05(\mathrm{ppm})^{-1} \\
& +\mathrm{C}_{\text {Ethyl Benzene }}(\mathrm{ppm}) \times 1.09 \mathrm{E}-02(\mathrm{ppm})^{-1} \\
& +\mathrm{C}_{\text {Tetrachloroethylene }}(\mathrm{ppm}) \times 1.76 \mathrm{E}-03(\mathrm{ppm})^{-1} \\
& +\mathrm{C}_{\text {Trichloroethylene }}(\mathrm{ppm}) \times 1.07 \mathrm{E}-02(\mathrm{ppm})^{-1}
\end{aligned}
$$

TABLE II: REFERENCE CONCENTRATION AND UNIT RISK FOR INDOOR AIR POLLUTANTS 


\begin{tabular}{|c|c|c|c|c|c|c|c|c|c|c|c|c|c|c|}
\hline Indoor air pollutant & 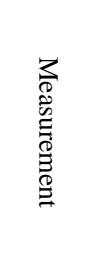 & $\stackrel{\text { S. }}{\Xi}$ & $\Xi$ & 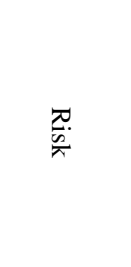 & 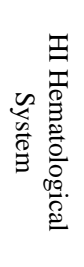 & 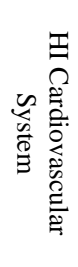 & 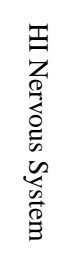 & 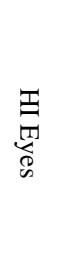 & 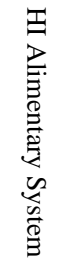 & 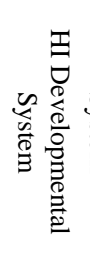 & 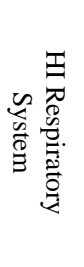 & 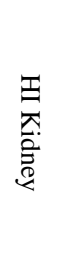 & 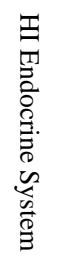 & 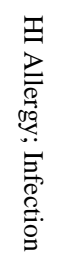 \\
\hline $\mathrm{CO}_{2}$ & 1,559 & ppm & 0.31 & & & & & & & & & & & \\
\hline $\mathrm{CO}$ & 6.8 & ppm & 0.76 & & & 0.76 & & & & & & & & \\
\hline $\mathrm{HCHO}$ & 0.27 & ppm & 36.84 & $1.99 \mathrm{E}-03$ & & & & & & & 36.84 & & & \\
\hline TVOC & 2.97 & ppm & & & & & & & & & & & & \\
\hline Benzene & 0.3644 & $\mathrm{ppm}$ & 19.40 & $6.98 \mathrm{E}-03$ & 19.40 & & 19.40 & & & 19.40 & & & & \\
\hline Carbon tetrachloride & & ppm & 0.00 & $0.00 \mathrm{E}+00$ & & & & & 0.00 & & & & & \\
\hline Chloroform & 0.9388 & ppm & 15.28 & $2.43 \mathrm{E}-02$ & & & & & 15.28 & 15.28 & & 15.28 & & \\
\hline 1,2-Dichlorobenzene & & ppm & & & & & & & & & & & & \\
\hline 1,4-Dichlorobenzene & 0.0242 & ppm & 0.18 & $1.60 \mathrm{E}-03$ & & & 0.18 & & 0.18 & & 0.18 & 0.18 & & \\
\hline Dichloromethane & & ppm & 0.00 & $0.00 \mathrm{E}+00$ & & & & & & & & & & \\
\hline Ethyl Benzene & 0.3016 & ppm & 0.65 & $3.27 \mathrm{E}-03$ & & & & & 0.65 & 0.65 & & 0.65 & 0.65 & \\
\hline Styrene & 0.1025 & ppm & 1.68 & & & & 1.68 & & & & & & & \\
\hline Tetrachloroethylene & & ppm & 0.00 & $0.00 \mathrm{E}+00$ & & & & & 0.00 & & & 0.00 & & \\
\hline Trichloroethylene & 0.0406 & ppm & 0.36 & 4.37E-04 & & & 0.00 & 0.36 & & & & & & \\
\hline Toluene & 0.9074 & ppm & 11.40 & & & & & & & & & & & \\
\hline Xylenes & 0.2904 & ppm & 5.40 & & & & 5.40 & & & & 5.40 & & & \\
\hline Bacteria & 2,935 & $\mathrm{CFU} / \mathrm{m}^{3}$ & 1.96 & & & & & & & & & & & 1.96 \\
\hline Fungi & 6,701 & $\mathrm{CFU} / \mathrm{m}^{3}$ & 6.70 & & & & & & & & & & & 6.70 \\
\hline $\mathrm{PM}_{10}$ & 184 & $\mu \mathrm{g} / \mathrm{m}^{3}$ & 2.45 & & & & & & & & 2.45 & & & \\
\hline $\mathrm{PM}_{2.5}$ & 13 & $\mu \mathrm{g} / \mathrm{m}^{3}$ & 0.37 & & & 0.37 & & & & & & & & \\
\hline $\mathrm{O}_{3}$ & 0.076 & $\mathrm{ppm}$ & 1.27 & & & & & 1.27 & & & 1.27 & & & \\
\hline Summation & & & $\underline{105.02}$ & $\underline{3.86 \mathrm{E}-02}$ & 19.40 & 1.13 & 26.67 & 1.63 & 16.12 & $\underline{35.34}$ & $\underline{46.15}$ & 16.12 & 0.65 & 8.66 \\
\hline
\end{tabular}

TABLE IV: HEALTH RISK ANALYSIS OF INDOOR AIR QUALITY FOR A LOCAL HOSPITAL (AFTER IMPROVEMENT)

\begin{tabular}{|c|c|c|c|c|c|c|c|c|c|c|c|c|c|c|}
\hline 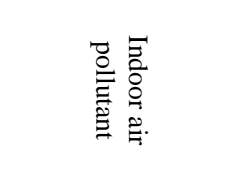 & 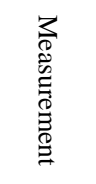 & $\stackrel{\Xi}{\Xi}$ & $\Xi$ & $\begin{array}{l}\frac{\pi}{\sigma} \\
\frac{n}{\pi}\end{array}$ & 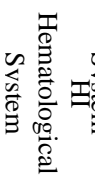 & 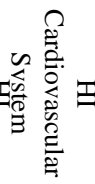 & 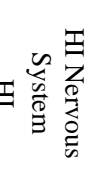 & $\underset{\infty}{\underset{\otimes}{\mathbb{N}}}$ & 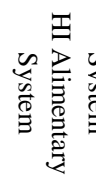 & 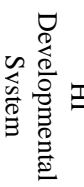 & 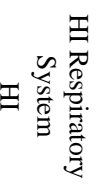 & 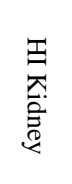 & 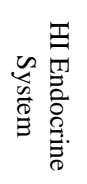 & 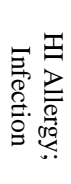 \\
\hline $\mathrm{CO}_{2}$ & 436 & ppm & 0.09 & & & & & & & & & & & \\
\hline $\mathrm{CO}$ & 0.32 & ppm & 0.04 & & & 0.04 & & & & & & & & \\
\hline $\mathrm{HCHO}$ & 0.01 & ppm & 1.36 & 7.37E-05 & & & & & & & 1.36 & & & \\
\hline TVOC & 0.01 & ppm & & & & & & & & & & & & \\
\hline Benzene & 0.0012 & ppm & 0.07 & $2.35 \mathrm{E}-05$ & 0.07 & & 0.07 & & & 0.07 & & & & \\
\hline Carbon tetrachloride & & ppm & 0.00 & $0.00 \mathrm{E}+00$ & & & & & 0.00 & & & & & \\
\hline Chloroform & 0.0032 & ppm & 0.05 & $8.18 \mathrm{E}-05$ & & & & & 0.05 & 0.05 & & 0.05 & & \\
\hline 1,2-Dichlorobenzene & & ppm & & & & & & & & & & & & \\
\hline 1,4-Dichlorobenzene & 0.0001 & ppm & 0.00 & $5.39 \mathrm{E}-06$ & & & 0.00 & & 0.00 & & 0.00 & 0.00 & & \\
\hline Dichloromethane & & ppm & 0.00 & $0.00 \mathrm{E}+00$ & & & & & & & & & & \\
\hline Ethyl Benzene & 0.0010 & ppm & 0.00 & $1.10 \mathrm{E}-05$ & & & & & 0.00 & 0.00 & & 0.00 & 0.00 & \\
\hline Styrene & 0.0003 & ppm & 0.01 & & & & 0.01 & & & & & & & \\
\hline Tetrachloroethylene & & ppm & 0.00 & $0.00 \mathrm{E}+00$ & & & & & 0.00 & & & 0.00 & & \\
\hline Trichloroethylene & 0.0001 & ppm & 0.00 & $1.47 \mathrm{E}-06$ & & & 0.00 & 0.00 & & & & & & \\
\hline Toluene & 0.0031 & ppm & 0.04 & & & & & & & & & & & \\
\hline Xylenes & 0.0010 & ppm & 0.02 & & & & 0.02 & & & & 0.02 & & & \\
\hline Bacteria & 277 & $\mathrm{CFU} / \mathrm{m}^{3}$ & 0.18 & & & & & & & & & & & 0.18 \\
\hline Fungi & 729 & $\mathrm{CFU} / \mathrm{m}^{3}$ & 0.73 & & & & & & & & & & & 0.73 \\
\hline $\mathrm{PM}_{10}$ & 6 & $\mu \mathrm{g} / \mathrm{m}^{3}$ & 0.08 & & & & & & & & 0.08 & & & \\
\hline
\end{tabular}




\begin{tabular}{|c|c|c|c|c|c|c|c|c|c|c|c|c|c|c|}
\hline $\mathrm{PM}_{2.5}$ & 1 & $\mu \mathrm{g} / \mathrm{m}^{3}$ & 0.03 & & & 0.03 & & & & & & & & \\
\hline $\mathrm{O}_{3}$ & 0.001 & ppm & 0.02 & & & & & 0.02 & & & 0.02 & & & \\
\hline Summation & & & $\underline{2.71}$ & $\underline{1.97 \mathrm{E}-04}$ & 0.07 & 0.06 & 0.09 & 0.02 & 0.05 & 0.12 & $\underline{1.48}$ & 0.05 & 0.00 & 0.91 \\
\hline Improvement & & & $97.4 \%$ & $99.5 \%$ & $99.7 \%$ & $94.3 \%$ & $99.7 \%$ & 98.9\% & $99.7 \%$ & $99.7 \%$ & $96.8 \%$ & $99.7 \%$ & $99.7 \%$ & $89.4 \%$ \\
\hline
\end{tabular}

\section{Case Study}

With the help of the Hospital IAQ Management Project, a local hospital intended to improve its IAQ in the first floor lobby, including the hanging area, leading pharmacies and waiting areas, aiming at ensuring the staff's and patients' health [8]. In 2009, the test results for the lobby IAQ are shown in Table III, which indicates carbon dioxide, formaldehyde, total volatile organic compounds, the total number of bacteria, fungi total number, $\mathrm{PM}_{10}$ and ozone concentrations exceeded the standard value. After counseling experts, experts suggest ways to improve it: (1) increasing the volume of fresh air intake in nighttime and daytime in order to reduce carbon dioxide and bacteria; (2) reducing personnel residence time in order to reduce the amount of bacteria and may also to improve the overall quality of health care; and (3) increasing the frequency of disinfection. Its effectiveness is as shown in Table IV.

\section{Discussion}

Before IAQ improvement, the HIs for hematological, cardiovascular, nervous systems, eyes, alimentary, developmental, respiratory systems, kidney, endocrine system, and allergy and infection are almost much higher than one; they are $19.40,1.13,26.67,1.63,16.12,35.34$, $46.15,16.12,0.65$ and 8.66, respectively. After IAQ improvement, the HIs for hematological, cardiovascular, nervous systems, eyes, alimentary, developmental, respiratory systems, kidney, endocrine system, and allergy and infection are largely reduced to $0.07,0.06,0.09,0.02$, $0.05,0.12,1.48,0.05,0.00$ and 0.91 , respectively. The overall $\mathrm{HI}$ reduced from 105.02 to 2.71 , up to $97.4 \%$. The cancer risk also reduced from $3.86 \mathrm{E}-02$ to $1.97 \mathrm{E}-04$, up to $99.5 \%$, but it is still unacceptable.

\section{CONCLUSION}

The difficulty in the study of analyzing health risk for indoor air quality is the determination of the reference levels, such as reference concentrations (RfC) for non-cancer risk and cancer unit risk (UR) for cancer risk. Therefore, this study proposed the method to define the reference levels using the most stringent values proposed by some environmental organizations, such as OEHHA, WHO, ACGIH, and Taiwan IAQ Standard. These organizations are selected because their reference levels are developed on a rigorously scientific basis. The target organ-specific hazard index (TOSHI) developed by USEPA was used here in order to clarify the HI and the associated affected target organs or systems. The case study has demonstrated the use of the proposed method. Future studies will extend this concept to deal with model specific diseases caused by indoor air pollutants based on epidemiology.

\section{ACKNOWLEDGMENT}

The authors would like to thank the National Science
Council of the Republic of China (Taiwan) for financially supporting this research under Contract NSC 101-2221-E-131-027.

\section{REFERENCES}

[1] Taiwan EPA, Taiwan Indoor Air Quality Act, 2011.

[2] OEHHA Air, "Air toxics hot spots risk assessment guidelines: technical support document for the derivation of noncancer reference exposure levels," 2008.

[3] WHO, WHO Human Health Risk Assessment Toolkit: Chemical Hazards, 2011.

[4] U.S. EPA, "National-scale air toxics assessment for 1996: draft for EPA science advisory board review," Biblio. Gov., 2013.

[5] California EPA. (2001). A guide to health risk assessment. [Online]. Available: http://www.oehha.ca.gov/pdf/HRSguide2001.pdf

[6] ACGIH, 2014 TLVs and BEIs, 2014.

[7] WHO, Air Quality Guidelines for Europe, 2000.

[8] R. K. Chen, "The effectiveness of improvement of indoor air quality for a local hospital," Technical Report of Chiali Hospital, 2010.

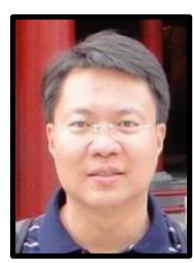

Kevin Liu received the Ph.D. degree in 1998 in civil engineering from National Central University, Taiwan. Currently, he is a professor in the Department of Safety, Health and Environmental Engineering, Ming Chi University of Technology, Taiwan. His research interests include the use of decision analysis and artificial intelligence techniques to environmental management issues.

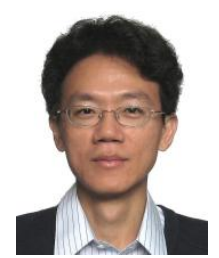

Ken Yeh received the M.S. degree in 1990 and the Ph.D. degree in 2000 both in civil engineering from National Central University, Taiwan. Currently, he is an associate professor in the Department of Construction Science and Technology, De-Lin Institute of Technology, Taiwan. His research interests lie generally in modeling and simulation of dynamics systems, and use of fuzzy logic techniques.

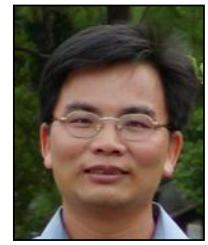

Ming-Jui Hung received the Ph.D. degree in 1999 in civil engineering from National Central University, Taiwan. Currently, he is an assistant professor in the Department of Safety, Health and Environmental Engineering, Ming Chi University of Technology, Taiwan. His research interests include indoor air quality, UVGI technique, study of green building materials.

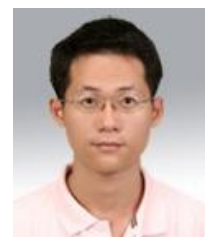

Cheng-Wu Chen is currently a professor at the Institute of Maritime Information andTechnology, National Kaohsiung Marine University. He received the B.S. degree in harbor and river engineering from National Taiwan Ocean University, Keelung,Taiwan, in 1999, the two Ph.D. degrees in civil engineering from National Central University in 2004 and information management from National Kaohsiung First University of Science and Technology in 2011. His research and teaching interests are in the area of applications of artificial intelligence, informationtechnology \& system, risk analysis \& management, logistics, decisionsupport, simulation optimization, GIS \& RS, and system control etc. forengineering and management problem solving.

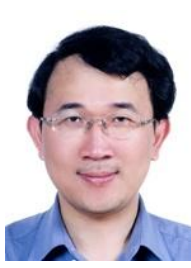

Yung-Shuen Shen received the Ph.D. degree in 1996 in chemical engineering from National Taiwan University of Science and Technology, Taiwan. Currently, he is an associate professor in the Holistic Education Center, Mackay Medical College, Taiwan. His research interests include incorporate environmental management and GHG issues. 